\title{
Optical configuration and color-representation range of a variable-pitch dot matrix holographic printer
}

\author{
Chih-Kung Lee, Jeremy Wen-Jong Wu, Sheng-Lie Yeh, Chih-Wen Tu, Yi-An Han, \\ Eric Hong-Zong Liao, Linus Ying-Yueh Chang, I-En Tsai, Hsiu-Hung Lin, \\ Jeffrey Chi-Tang Hsieh, and Julie Tsai-Wei Lee
}

\begin{abstract}
The optical system configuration and design of a dot matrix holographic printer that can create image grating pixels of variable size, arbitrary pitch, and discretionary angle on a photoresist plate are presented. With the capability to vary spot size, grating orientation, and grating pitch on the fly, this newly developed holographic printer can apply a prespecified color at each specific viewing angle. Diffractive images with various visual effects and the wide color range that are possible by use of this system are examined in detail. (c) 2000 Optical Society of America

OCIS codes: $\quad 090.1760,120.4640,090.4220,050.1950,120.4570,350.2770$.
\end{abstract}

\section{Introduction}

It has long been an objective of many researchers to create holograms ${ }^{1-3}$ with the assistance of computers. ${ }^{4-15}$ Dot matrix holograms, the products of a unique type of computer-generated holography that uses many small dots consisting of diffraction gratings upon a flat or curved surface to create a holographic image pixel by pixel as today's computer printers do, have been gaining in popularity as the technology continues to advance. Because of the similarity of dot matrix hologram mastering machines and today's printers, these type of machine are generally called dot matrix holographic printers or dot matrix mastering origination systems. A dot matrix holographic printer records the interference fringes of two laser beams to form small dots called grating pixels. A dot matrix hologram thus has a unique appearance, as its image is made up of grating pixels that are visible under a microscope. To achieve this form of presentation efficiently, dot matrix holographic printers use a computer to determine the characteristics of each grating pixel and then

C.-K. Lee (cklee@mems.iam.ntu.edu.tw), J. W.-J. Wu, C.-W. Tu, Y.-A. Han, and E. H.-Z. Liao are with the Institute of Applied Mechanics, National Taiwan University, Taipei 106, Taiwan. S.-L. Yeh, L. Y.-Y. Chang, I.-E. Tsai, H.-H. Lin, J. C.-T. Hsieh, and J. T.-W. Lee are with AHEAD Optoelectronics, Inc., No. 13, Chin-ho Road, Chung-ho, Taipei hsien 235, Taiwan.

Received 26 April 1999.

0003-6935/00/010040-14\$15.00/0

(C) 2000 Optical Society of America download the corresponding processing parameters to control the optomechanical configuration within the holographic printer to create a dot matrix master upon a photoresist plate. The arrangements, layouts, and shapes of these dots can be used to distribute incoming laser beam power to specific locations. These features can be combined for purposes such as anticounterfeiting applications of products and documents. Dot matrix holograms are also unique in that they are able to combine dynamic or kinetic and three-dimensional effects to create striking images that are easily viewable under normal lighting. In general, it is difficult to use traditional holographic printers to create true-color holograms effectively because it is not possible to vary the pitch of each grating pixel. To further advance the application range of dot matrix holograms, efficient color control must be introduced into the dot matrix hologram origination process. Here we report the optical, mechanical, data-flow configuration, and color-representation range of a newly developed holographic printer named Sparkle that possesses the capability to specify the pitch within each grating pixel.

In the literature, few peer-reviewed papers that treat dot matrix holographic printers are available. However, a total of five U.S. patents issued to date are known to be directly related to dot matrix holography. ${ }^{16-20}$ One can see by examining these patents in chronological order that color control has been the primary focus of dot matrix holograms since their inception. The first patent ${ }^{16}$ does not deal with a particular system configuration but instead focuses on how to create the data flow and data structure 


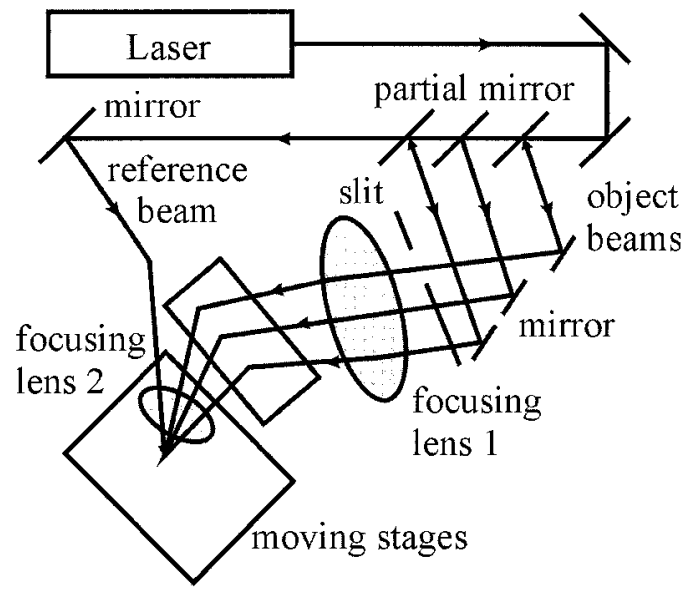

Fig. 1. Optical layout from the Toppan patent. ${ }^{17}$

needed to expose many diffraction grating pixels. In addition, the use of curved grating lines within a grating pixel to create a gray-scale image is clearly described. The second patent ${ }^{17}$ details ways to input and use diffraction gratings to represent many two-dimensional images. An optical configuration in this patent, shown in Fig. 1, a configuration that can be used to control color of dot matrix holograms is shown. The incident laser light beam is partially split into several object beams and a reference beam. The interference among the reference beam and the object beams is then used to form grating pixels. The patent details the way in which one can use the pitch of each grating pixel to determine the color of that grating pixel under specific lighting and observation conditions. Variation of the grating pitch is achieved by determination of the object beam that is to be selected by the slit.

A slightly different optical configuration (Fig. 2) found in the second patent ${ }^{18}$ uses four beams to control the color within each pixel of a dot matrix hologram. Figure 3 shows the optical configuration described in another patent. ${ }^{19}$ Here the beam-

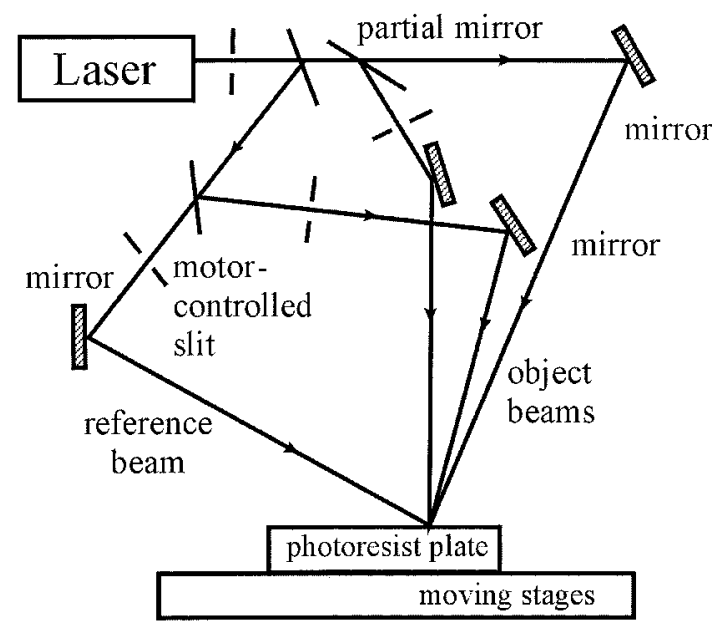

Fig. 2. Optical layout from the first Davis patent. ${ }^{18}$

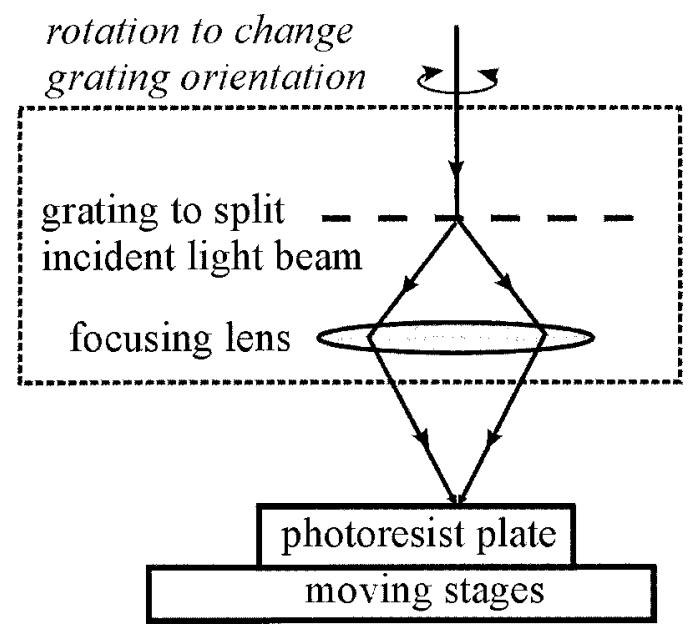

Fig. 3. Schematic of Applied Holographic's patent. ${ }^{19}$

splitting grating and lens are fixed inside a rotational stage (Fig. 3). When the laser beam source goes through the beam-splitting grating, only the +1 and the -1 orders of the diffraction beams will be selected to pass through the focusing lens. Then these two beams will converge to form an interference spot upon the photoresist plate. The fifth patent, ${ }^{20}$ issued in October 1998, is the latest patent of which we aware that is directly related to dot matrix holographic technology. This patent, whose original disclosure was filed in 1988, with a continuation-in-part application filed in the fall of 1993 and a further continuation in May 1996, discloses a series of optical configurations that can be used to generate dot matrix holograms. In all these systems a laser beam is split into a reference beam and at least one object beam. An example of the optical configuration of these systems is shown in Fig. 4. Modulation of at least one object beam and adjustment of the angle at which that beam interferes with the reference beam on the photoresist plate create the grating pixels. From Fig. 4 it is clear that the viewing angle and the color distributed by each of the grating pixel are functions of the partial mirrors and of the mirrors that are used to guide the object beams.

It is clear from a review of these five dot matrix hologram-related patents that the salient capability of a dot matrix hologram is its ability to control the grating pitch and thus to control the distribution of color beams. This color control methodology uses two coherent laser beams to generate a grating image pixel by pixel (Fig. 5). Despite the color-control origin of the dot matrix hologram, most commercially available dot matrix master origination systems developed so far lack a simple and effective approach to control the grating pitch on the fly because of the limitations of the optical configurations adopted. The dot matrix holographic printer system reported here can vary the grating pitch of each grating pixel easily and effectively as a result of a new optical configuration that is used. ${ }^{21}$ The fundamental de- 


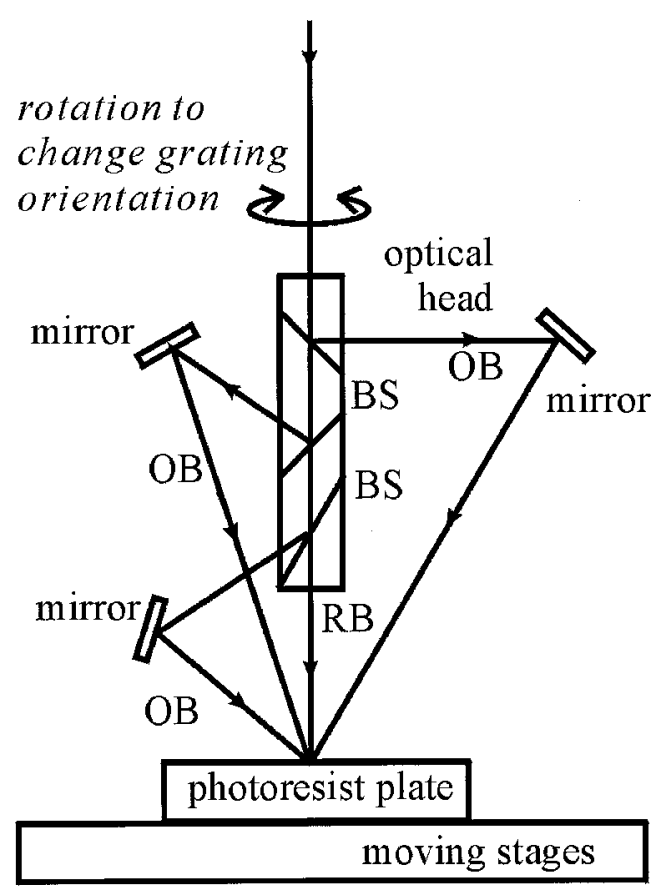

Fig. 4. Optical layout from the second Davis patent ${ }^{20}$ : OB's, object beams; RB, reference beam; BS's, beam splitters.

sign concept of this innovative optical configuration is similar to the optical configuration adopted in today's laser Doppler anemometer systems. ${ }^{22}$

It can be seen that two parameters (Fig. 5) determine the characteristics of each grating pixel within a dot matrix hologram. The first parameter is the grating pitch, $d$, which is determined by the intersection angle, $\theta$, of the two incoming coherent laser light beams. The larger the intersection angle, the smaller the grating pitch. More specifically, grating pitch $d$ equals $\lambda / 2 \sin (\theta / 2)$, where $\lambda$ is the wavelength of the incoming laser beams. The second parameter is the grating orientation, which is determined by the direction of the two incoming laser beams. More specifically, the holographic printers create all dot matrix holograms pixel by pixel by varying grating

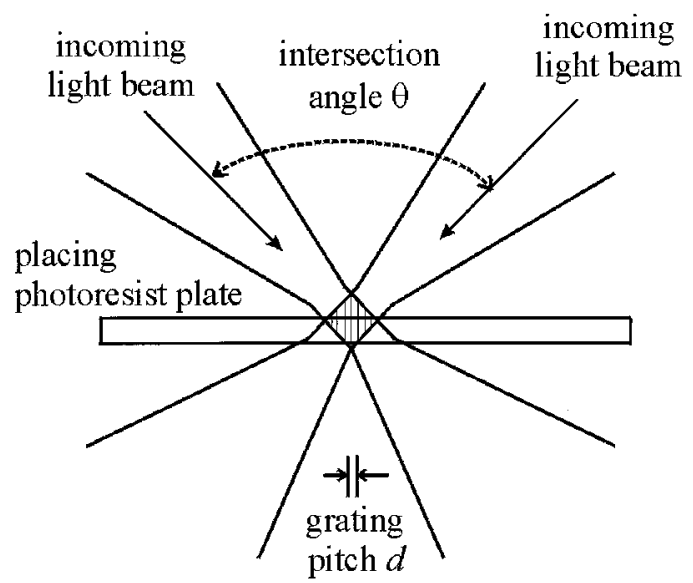

Fig. 5. Spot grating image created by two focused beams. pitch $d$ and the grating orientation of each grating pixel. All commercially available dot matrix holographic printers use the interference pattern generated by two laser light beams to create each grating pixel, as shown in Fig. 1. During normal operations, a photoresist plate is placed in an appropriate location to record the interference patterns.

As the fundamental concept of dot matrix holograms lies in writing the image pixel by pixel by varying the grating pitch and grating orientation, there are potentially many other approaches that can be used to create similar types of dot matrix hologram. In addition to the methodology that uses two interference beams, shown in Figs. 1-5, another common approach is to apply laser-beam or $e$-beam lithography ${ }^{23-27}$ to create each grating pixel. As laser-beam or $e$-beam lithography is commonly used in making photoresist masks for today's semiconductor processing, the equipment is widely available within the semiconductor processing industry. Dot matrix holograms generated by laser-beam or $e$-beam lithography are commonly referred to as $e$-beam holograms, or kinegrams. ${ }^{27,28}$ Inasmuch as laserbeam or $e$-beam lithography can specify the linewidth, line orientation, and line location of every pixel, a dot matrix hologram created by $e$-beam lithography can generally achieve even better resolution and brightness. However, the data files needed to generate a laser-beam or $e$-beam hologram are typically in the range of gigabytes..$^{29,30}$ In addition, using a laser writer or $e$-beam machine is extremely expensive. A standard $e$-beam machine can cost more than $\$ 10$ million. This is the main reason that $e$-beam lithography is not readily available; generally only researchers or hologram manufacturing companies that belong to large consortiums or organizations that owns or have easy access to $e$-beam machines can produce $e$-beam holograms. For example, the holographic team within Dai Nippon Printing Company, Ltd., ${ }^{25}$ of Japan has performed extensive research on $e$-beam holograms, it has an $e$-beam machine in house. The research team formed by National Taiwan University and AHEAD Optoelectronics, Inc., which also has easy access to $e$-beam machines at the facilities of Taiwan's booming semiconductor industry as well as the National Nano Device Laboratory, ${ }^{31}$ has also developed an $e$-beam holography program, called Radiant. This program is an add-on option to Sparkle and is used mainly to facilitate the implementation of $e$-beam holography. Making an $e$-beam hologram requires a GDS-II data file format, which an $e$-beam machine can read. For example, the Radiant program accompanied by the translation program within Sparkle can translate the bit map output of many MS Windows-based design packages such as CorelDraw and Photoshop into a GDS-II data file. Following creation of the photolithography mask by the laser writer or the $e$-beam machine, standard semiconductor processing equipment called a mask aligner is introduced into the fabrication process. An aligner has an UV light source and a set of optics that can transform the 


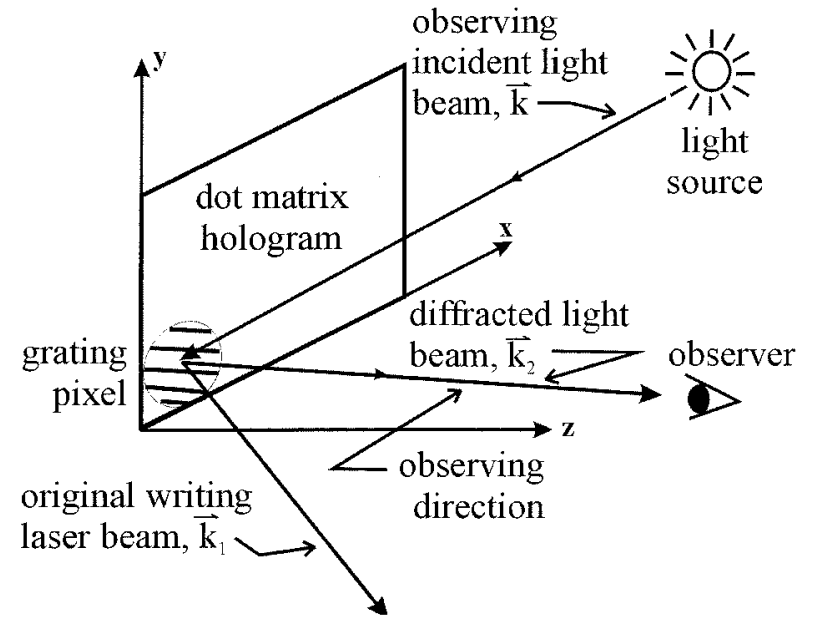

Fig. 6. Grating pixel diffracting incident beam 1 into an observing direction.

photolithography mask into a pattern on a photoresist plate, which is the $e$-beam or laser-beam hologram master. Once this hologram master is created, the traditional hologram manufacturing process including metallizing, electroforming, mechanical recombining, and embossing can be used to mass-produce the holograms. The above descriptions demonstrate the close ties between dot matrix holography and $e$-beam or laser-beam holography. With the wide discrepancies between the system cost of dot matrix holography and $e$-beam or laser-beam holography, it is expected that dot matrix holography will gain even wider acceptance, provided that an effective and versatile dot matrix holographic printer can be developed. It hoped that the system detailed in this paper will serve as a triggering point for an even more technically advanced dot matrix holographic mastering system.

\section{Theory}

The underlying philosophy of the design process for dot matrix holograms is to create a graphic filter that converts the original graphic design based on a series of grating pixels. We know that all grating pixels can diffract a specific wavelength of incident light to the eye of an observer by means of the gratings, which in turn allow the observer to see light diffracted from the grating of each grating pixel in the hologram at a specific angle. The integration of all the grating pixels in the hologram then generates the effects required for the original graphic design. If we want to diffract the incident light to a particular direction, we can start by recording the interference fringes of the incident light beam and a light beam traveling along the observing direction. More specifically, a light beam incident from one direction of the original two incident light beams that form the interference fringes will be diffracted to the direction of the other original recording beam. Figure 6 illustrates how a grating pixel is formed by the recording fringes from

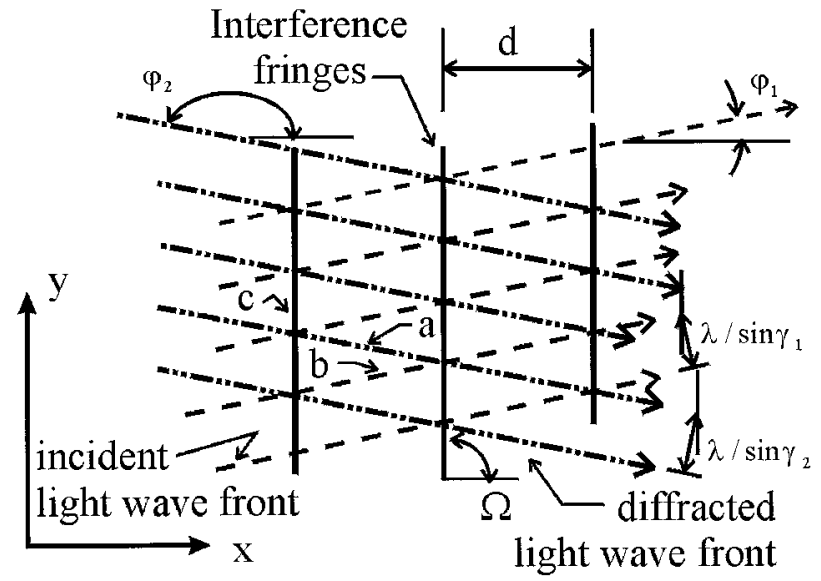

Fig. 7. Geometric relationship to determine grating pitch and grating orientation.

incident light beams $\mathbf{k}_{1}$ and $\mathbf{k}_{2}$. When observing incident light beam $\mathbf{k}$ is incident onto the grating pixel that is coated with a layer of reflective material, light beam $\mathbf{k}_{2}$ can be rebuilt by diffraction.

Based on the concept of grating images, it is obvious that the original writing light beams, an incident observing light source, diffractive light beams, and pitch as well as orientation of the grating pixels are the main factors that influence the reconstruction of the desired images. For illustration, $\mathbf{k}$ is the direction of the observing incident light beam, $\mathbf{k}_{2}$ is the direction of the diffracted light beam as well as of one of the original writing laser beams, and $\mathbf{k}_{1}$ is the direction of the other original writing laser beam. For the case of a typical dot matrix hologram in which a reflective coating was coated on top of a grating to create a reflective image as shown in Fig. 6, $\mathbf{k}$ is the mirror image of $\mathbf{k}_{1}$ with respect to the plane where the grating pixel is located. Assuming that the directional cosine of $\mathbf{k}_{1}$ is $\left(\cos \alpha_{1}, \cos \beta_{1}, \cos \gamma_{1}\right)$ and that the directional cosine of $\mathbf{k}_{2}$ is $\left(\cos \alpha_{2}, \cos \beta_{2}, \cos \gamma_{2}\right)$, the wave front of one of the original writing light beam will determine a family of plane $\mathrm{K} 1$, which has a normal vector $\mathbf{k}_{1}$. The intersecting lines of $K 1$ and the $x-y$ plane where the hologram is located can be formulated as $\cos \alpha_{1} x+\cos \beta_{1} y=d$, which denotes a series of parallel lines with slope $-\cos \alpha_{1} / \cos \beta_{1}$, pitch distance $\lambda / \sin \gamma_{1}$, and an intersecting angle with its $x$ axis equal to $\varphi_{1}=\tan ^{-1}\left(\cos \beta_{1} / \cos \alpha_{1}\right)+$ $\pi / 2$ (Fig. 7). Similarly, the intersecting lines related to diffractive light $\mathbf{k}_{2}$ are of slope $-\cos \alpha_{2} / \cos \beta_{2}$ and pitch distance $\lambda / \sin \gamma_{2}$ and have an intersecting angle with the $x$ axis equal to $\varphi_{2}=\tan ^{-1}\left(\cos \beta_{2} / \cos \alpha_{2}\right)$ $+\pi / 2$ (Fig. 7). From basic holographic principles, we know that the two series of parallel lines will produce another series of parallel lines that represent interference fringes with pitch $d$ and orientation $\Omega$. The three edges which are designated $a, b$, and $c$ in Fig. 7, denote the wave front of the incident light, the wave front of the diffractive light, and the interfer- 
ence fringes, respectively. Considering the cosine relation in geometry, we can express pitch $d$ and orientation $\Omega$ of the gratings as

$$
\begin{aligned}
& \Omega=\varphi_{1}-\sin ^{-1}\left(\frac{\lambda}{c \sin \gamma_{1}}\right), \\
& d=a \sin \left(\varphi_{2}-\Omega\right),
\end{aligned}
$$

where $a=\left(\lambda / \sin \gamma_{1}\right)\left[1 / \sin \left(\pi+\varphi_{1}-\varphi_{2}\right)\right], b=(\lambda / \sin$ $\left.\gamma_{2}\right)\left[1 / \sin \left(\pi+\varphi_{1}-\varphi_{2}\right)\right]$, and $c^{2}=a^{2}+b^{2}-2 a b$ $\cos \left(\pi+\varphi_{1}-\varphi_{2}\right)$.

In a grating image design concept, pitch $d$ and orientation $\Omega$ of each grating pixel are the most fundamental parameters. They determine how the incident light beam can be diffracted precisely to where the observer is and thus can be used to rebuild the desired image according to the originally intended effects for each grating pixel. From Eqs. (1) and (2) it is clear that the orientation of each grating pixel is a function of the position of the light source, the observing location, and the position of each grating pixel. At the same time, the grating pitch of each grating pixel is influenced by four parameters: the position of the light source, the observing location, the grating pixel position, and the color wavelength of each grating pixel. Thus a graphic designer needs first to decide the location of a fixed light source, the position of the observer, the color distribution, and the desired viewing effects of every graphic pixel of the original graphic design. For a dot matrix program to have the correct preview function, all the information mentioned above must be incorporated into the design program that calculates the pitch and orientation of each graphic pixel of the desired graphic image. Note that a grating pixel can diffract the incident light beam only into rainbow colors. True-color dot matrix holograms require adoption of a color-addition process. In other words, more than one grating pixel must be combined to form a graphic pixel to create true-color dot matrix holograms.

As every grating pixel on a dot matrix hologram is actually a simple grating, the visual angle for each pixel is small. However, this small-visual-angle phenomenon translates into a bright pixel when the observer is located within the designated viewing angle for that specific pixel, as all the light energy is concentrated within that small angle. As the computed diffraction angles are varied, the grating on each pixel will vary accordingly. Light beams of different wavelengths, i.e., different colors, will then be diffracted to a corresponding observing position. In other words, the observer will see a different color for a specific grating pixel. When the wavelength of the light beam that was used to diffract the incident light beam into a predesigned angle exceeds the range of visible light, the observer will not be able to see that specific grating pixel for which a white-light source was used. By calculating the specific grating pitch and orientation one can adapt this fundamental concept to create special effects such as zoom-in, zoomout, or changing shape.

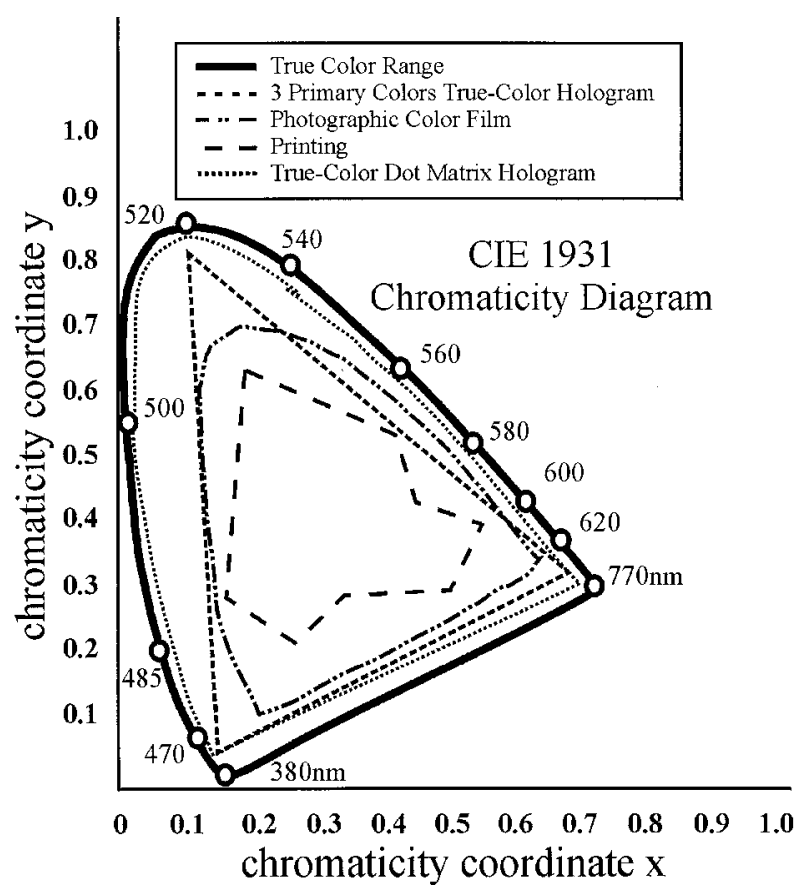

Fig. 8. 1931 chromaticity diagram showing the color ranges of various methods.

\section{Color Reconstruction}

Color-reproduction technology is one of the most important techniques used in printing, computer monitors, television monitors, color printers, and color films. The major color reproduction method used in printing is the CYMK (cyan, yellow, magenta, black) system; the RGB (red, green, blue) system is used for computer monitors and color film technology. ${ }^{32-34}$ More specifically, both color methods separate images by using three predetermined primary colors (i.e., CYM or RGB) and then subtract or add color to reproduce the color of the original image. Unfortunately, these two methods are not able to reconstruct all the colors seen by the human eye, especially metallic colors. It will be shown below that true-color dot matrix holograms can encompass a much wider color range than the more traditional colorreproduction approaches mentioned above.

The Commission Internationale de l'Eclairge (CIE) chromaticity diagram, which was developed to describe how object colors are perceived by a typical observer in various lighting conditions, can be used to overcome the difficulty mentioned above. In the 1931 CIE chromaticity diagram (Fig. 8) the two chromaticity coordinates $x$ and $y$ can be used to describe the location of the various colors. The inner region of the half-elliptically shaped curve in Fig. 8 represents all the visible colors that can be perceived by the human eye. The boundary of this half-ellipse represents pure colors, which are formed by narrowbandwidth light beams. These boundary points have roles similar to those of the primary colors RGB or CYM of the color-separation systems. More specifically, the primary colors of RGB or CYM are in fact 
vertex points located within the true-color range. It should be noted that all the colors encompassed by a region that is defined by a series of vertex points can be represented by proper choice the relative intensities of the colors represented by these vertex points. Using this concept, we can identify the maximum color range obtainable from conventional printing technology by examining the location of the primary colors (CYMK) of the traditional four-color printing method. Similarly, we can also examine the color range that is accessible by a television set by measuring the chromaticity coordinates (i.e., chroma and hue) of the three primary colors (RGB) of television monitor pixels. More specifically, true-color holograms of the traditional method (Fig. 8) use three primary colors to form the true colors observed in the hologram. This process indicates that traditional true-color holograms can display only colors located within the region encompassed by these three extreme points. The color region that can be displayed by a photographic color film and by a conventional printing method are also shown in Fig. 8 to permit the reader to compare the color regions obtainable for the various color-reconstruction processes. The wider the color region, the more powerful the colorreconstruction processes.

Compared with the color-separation models such as RGB, CYMK, and HGB (hue, saturation, and brightness), the CIE color model has the advantage of quantification and linear superposition. More specifically, the CIE color model can completely cover all visible colors and can precisely describe color coordinates as hue and chroma (Fig. 8). In addition, with this CIE color model we can use a linear superposition to convert any color into different ratios of the primary colors. It is clear that a traditional truecolor hologram reconstructs the object color by adding the three primary colors RGB. In other words, a color-addition process is used to re-create colors in the hologram. This process is completely different from the color subtraction that is used in the printing process. If we can add more color components into a hologram-making process, the color range that a true-color hologram can display should be in fact much wider than that of the conventional printing process. It is shown below that the chromaticity coordinates of various grating pixels created with Sparkle and illuminated by various light sources can cover almost the entire domain of the CIE color model (Fig. 8). That is, combining many grating pixels of different pitches to form a graphic pixel can produce truecolor dot matrix holograms that have the widest color-display region of any color-reconstruction scheme.

\section{Implementation Methodologies}

Dot matrix holographic mastering technology is continually being improved. Limits are being pushed toward higher resolution, faster speed, increased security features, better fidelity to vivid true colors, smaller overall machine size, and lower cost. Some traditional dot matrix holograms, which are typically

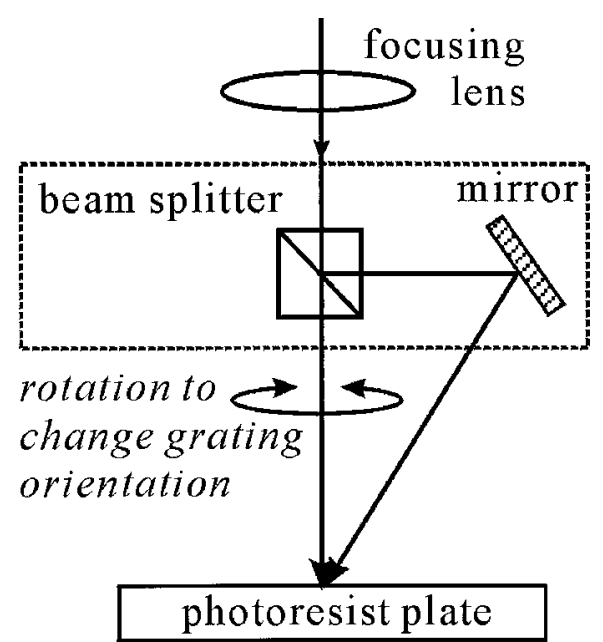

Fig. 9. Schematic of an asymmetric two-beam interference holographic printer.

of lower resolution, can achieve many different spot shapes by simply changing the incident beam shapes. However, in today's high-resolution dot matrix holograms, say, higher than 600 dots per inch (dpi), the spot shape is typically round because of focusing.

Some of the technical advancements in dot matrix holograms during the past few years are reviewed herein. The configuration of a commercially available dot matrix holographic printer, which is slightly different from the Davis's printer ${ }^{18,20}$ described above because it possesses only one reference light beam and one object light beam instead of one reference light beam and three object light beams, is shown in Fig. 9. In this two-beam interference holographic printer (Fig. 9) the laser beam goes through the focusing lens and then is split into a converged reference beam and a converged object beam. The converged object beam reflected by the mirror intersects the reference beam to form a small grating pixel upon the photoresist plate. Both the beam splitter and the mirror are fixed upon a rotational stage. We can alter the orientation of the gratings and the size of each grating pixel recorded on the photoresist plate by rotating the stage and by moving the lens, respectively. Two drawbacks exist in this type of asymmetric optical configuration. One is that the effective grating area is smaller than that of the corresponding spot [Fig. 10(a)] because of the different optical path lengths and the different incident angles of the two interference light beams. The second is that it is difficult to alter the grating pitch on the fly during the recording process. The first drawback lowers the diffraction efficiency and increases the noise. The second drawback allows a graphic designer to specify only one color when the image is viewed along a specific direction, which then prevents the creation of true-color dot matrix holograms.

To neutralize the first drawback, we consider the systems shown in Figs. 3 and 11. In the symmetric optical configuration shown in Fig. 11 the beam splitter, the mirrors, and the lenses are all fixed inside a 


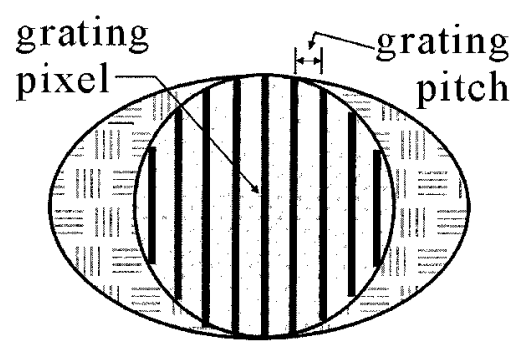

(a)

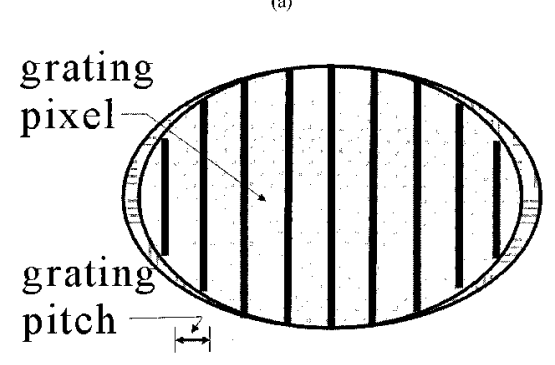

(b)

Fig. 10. Shape of an effective grating pixel in (a) a asymmetric and (b) a symmetric optical configurations.

rotational stage. The laser beam source is first split into two light beams by the beam splitter. The two laser beams are reflected by mirrors and then focused by a lens. These two laser beams finally intersect and form a grating spot upon the surface of the photoresist plate. In the symmetric configurations as shown in Figs. 3 and 11 the efficiency of the grating pixel can be improved, as the two laser beams will, in theory, completely overlap [Fig. 10(b)]. However, neither of these two configurations can be used easily to circumvent the second drawback that we mentioned. To achieve the goal of altering the grating pitch within each grating pixel, we must change the intersecting angle between the two interfering laser beams. The main difficulty in designing and constructing a system that can change the grating pitch on the fly lies on the fact that the focusing spot will

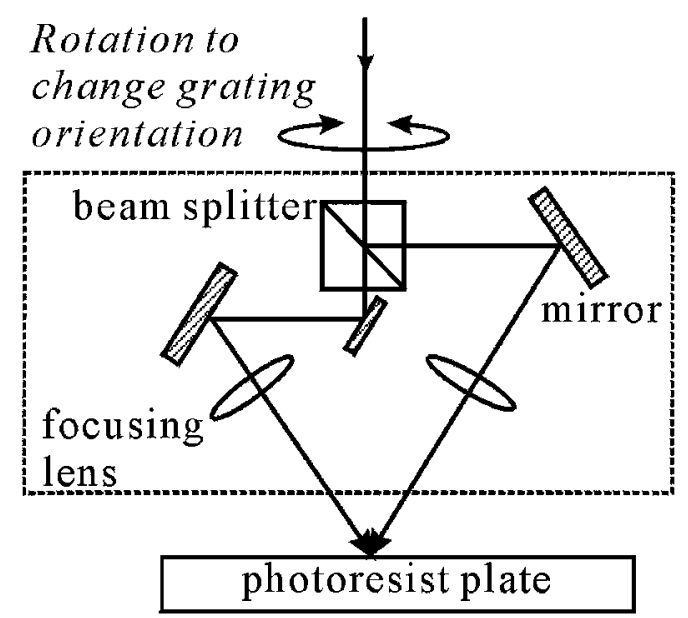

Fig. 11. Schematic of another two-beam interference holographic printer.

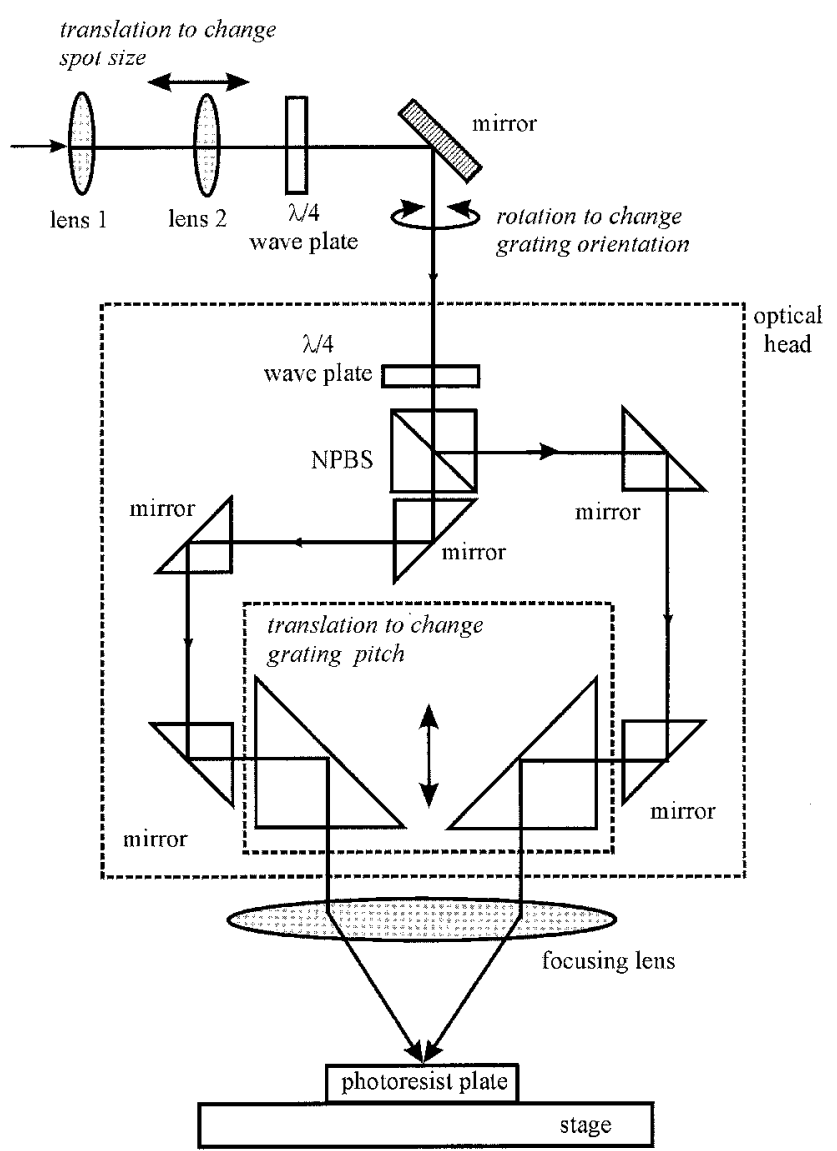

Fig. 12. Schematic of the newly developed two-beam interference holographic printer Sparkle.

move in and out of the photoresist plate whenever the intersection angle of the two recording laser beams is changed. Without the proper design, the approach needed to combat focusing spot location variations can make a dot matrix machine complex and difficult to align.

The newly developed optical configuration that maintains the advantages of symmetric design and permits easy pitch variation is shown in Fig. 12. The linearly polarized incident laser beam generated by a He-Cd laser operating at a $442-\mathrm{nm}$ wavelength is converted into a circularly polarized light beam by the first quarter-wave plate. The second quarterwave plate transforms the circularly polarized laser beam into a linearly polarized light beam that is polarized $45^{\circ}$ with respect to the nonpolarizing beam splitter (NPBS). This arrangement guarantees that the polarization states of the two laser beams split by the nonpolarizing beam splitter will be identical and remain $s$ polarized with respect to all reflective mirrors within the optical head when the optical head is rotated to control grating pixel orientation. After the incident laser beam is split by the beam splitter into two incident laser beams, the two coherent light beams are focused onto the photoresist plate by a specially designed focusing lens. Grating pitch $d$ of the grating pixel formed upon the photoresist plate 


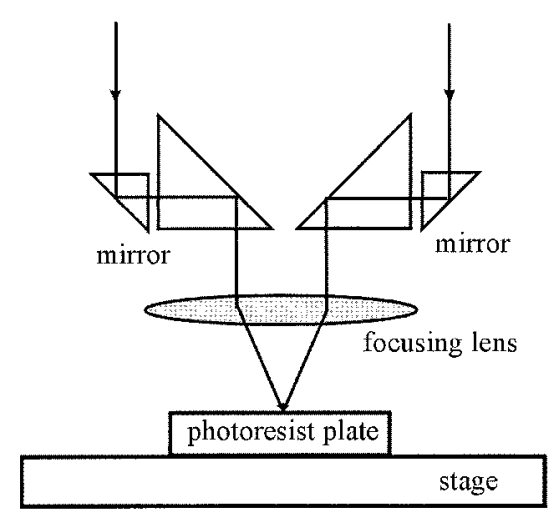

(a)

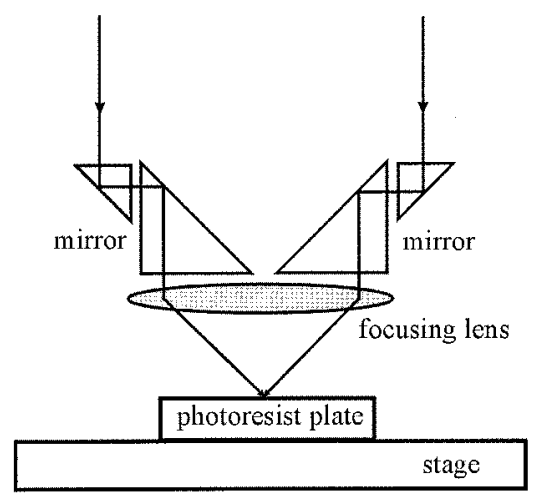

(b)

Fig. 13. Changing grating pitch on the fly with the newly developed Sparkle printer.

can be varied on the fly by translation of a stage shown in Fig. 12 to change the distance of the two coherent light beams before they impinge upon the focusing lens. All mirrors in the optical head can be shown to operate in a total-internal-reflection mode in Fig. 12 even though the first surface laser mirrors can serve the same purpose. Noted that the totalinternal-reflection mirror was used in the configuration shown in Fig. 12, so the system can operate at a wide variety of wavelengths without the need to change many optical components. There are several design features worth mentioning in the optical configuration shown in Fig. 12. First, this design abandoned the concept of using a reference and object light beams as seen in Figs. 1, 2, 4, and 9 and instead uses two identical light beams. Second, even though the implementation adopted for Fig. 12 is similar to that of a typical interferometer, the two laser light beams that form the final grating pixel need not have identical intensities because of the recording characteristics of the photoresist used. Our experience has shown that, as long as the intensity ratio between the two light beams remains $1: 10$, the final grating results will be quite acceptable for the typical photoresist used for the chosen laser wavelength.

As shown in Fig. 13, the larger the distance between the two coherent light beams, the smaller the

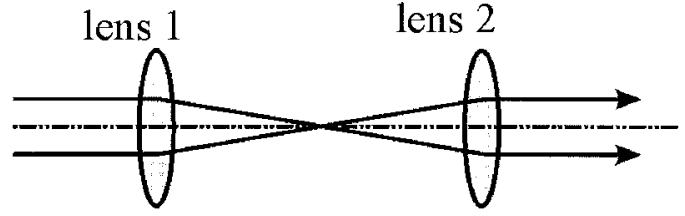

(a)

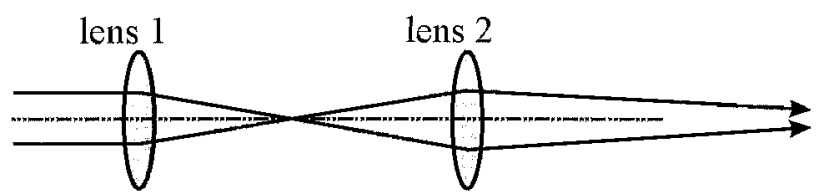

Fig. 14. Changing the light beam convergent angles on the fly by use of a lens pair.

grating pitch $d$. The focusing lens shown in Figs. 12 and 13 was especially designed and fabricated to prevent spherical aberration of this focusing lens from changing the location of the focusing spot whenever the distance between the two incoming coherent laser beams is changed. Varying the spot size can change the relative diffraction efficiency of each grating pixel, which is an important considering in creating true-color dot matrix holograms. The spot size control implemented in Sparkle is shown in Figs. 14 and 15. If the back focal plane of lens 1 coincides with the front focal plane of lens 2 , a collimated light beam on entering lens 1 will remain collimated after it passes through lens 2 [Fig. 14(a)]. This configuration will achieve the smallest focusing spot. If lens 2 of Fig. 14(b) is moved farther from the back focal plane of lens 1 , the transmitted light beam after lens 2 will converge. This converged light beam will be

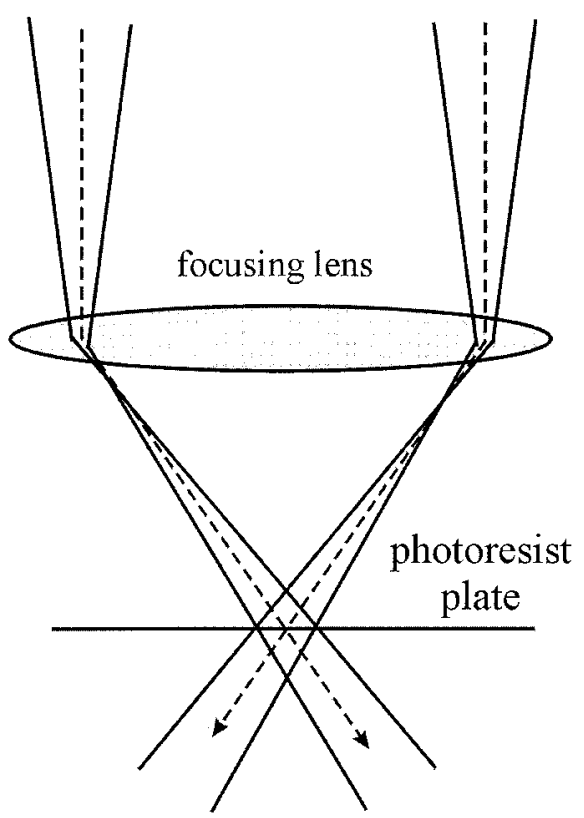

Fig. 15. Changing the grating pixel spot size on the fly with the newly developed Sparkle printer. 
further converged by the focusing lens, as shown in Fig. 15. It is clear from the above discussion that the spot size will depend on the converged angle of the transmitted light beams of lens 2 shown in Fig. 14. This is the mechanism adopted in Sparkle to modify the spot size, which is then used to control the relative diffraction efficiency needed to create truecolor dot matrix holograms. Note that spot size is independent of the grating pitch in the configuration shown in Fig. 12, an important consideration for creating vivid true colors. In addition, as these two converged light beams that were used to change the spot size (Fig. 14) remain parallel to the optical axis of the focusing lens, they will continue to intersect at the focal point of the focusing lens that coincides with the photoresist plate. In addition, the spot shape will remain circular for the configuration shown in Fig. 14(b). This circular spot shape will be easier to arrange than the elliptical spot shape shown in Fig. 10(b). Furthermore, as the wave fronts of the two converged light beams are basically spherical, the interference fringes formed by these two convergent spherical wavefronts will remain a series of straight lines. This property provides us with a chance to use the spot size to control the diffractive efficiency of each grating pixel. As the spot size obtained from the configuration shown in Fig. 14(b) will be larger than that of the configuration shown in Fig. 14(a), it will achieve a higher relative intensity to represent the specific color. By varying the relative sizes of all the grating pixels that form a particular color pixel, which displays a specific color that an observer can see, we can tailor the chromaticity coordinate of each graphic pixel.

It is clear from the above discussions that grating pixels generated by this newly developed holographic printer can have spot size, grating orientation, and grating pitch completely specified by the designer; i.e., the images constructed can show many prespecified colors at a specific viewing angle. Furthermore, the combined effects of controlling spot size, grating orientation, and grating pitch on the fly provides Sparkle with a capability to create true-color dot matrix holograms. Microscopic photos of the focusing spot at 400 and 800 dpi are shown in Fig. 16. It should be noted that, even in the same dpi configuration, Sparkle can still modify the relative diffractive efficiency of each grating pixel. The color-range argument represented by Fig. 8 further testifies to the advantages of the optical configuration adopted in Sparkle. The color range that can be encompassed by Sparkle will be examined below.

\section{Color Technology of Diffraction Gratings}

It can be seen from Fig. 8 that the borders of the CIE diagram are pure colors that represent pure-light wavelengths that range from 380 to $780 \mathrm{~nm}$. In addition, the newly developed dot matrix holographic printer Sparkle, which has an on-the-fly grating pitch-varying capability, can write grating pixels according to the user's design to diffract the appropriate light beams from the light source to the observer with

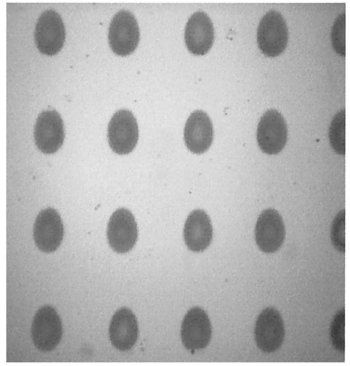

(a)

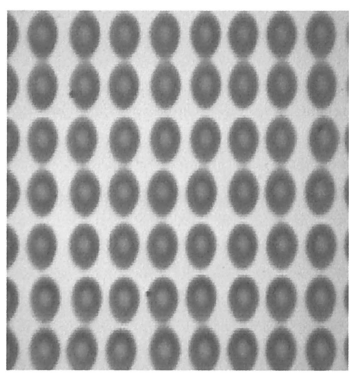

(c)

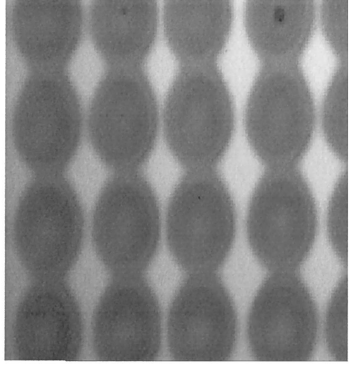

(b)

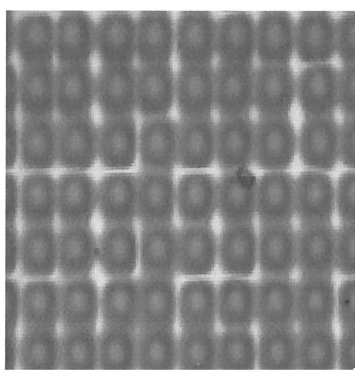

(d)
Fig. 16. Microscopic photos of grating pixels at different resolutions and at different spot sizes: (a) 400 dpi with small spot size, (b) 400 dpi with large spot size, (c) 800 dpi with small spot size, (d) 800 dpi with large spot size.

a nearly pure spectrum. In other words, the chromaticity coordinate of a grating pixel generated by this diffractive approach will be very close to the CIE diagram border. By selecting enough grating pixels from the borders of the CIE diagram and by using the linearity of the CIE color representation method, one can easily create almost any color located within the CIE color diagram by combining multiple grating pixels to form a graphic pixel. Any three grating pixels, which represent three different light wavelengths, can be chosen at one time to compose any color that happens to be located within the triangular area encompassed by the three color grating pixels (Fig. 17).

The basic equations related to defining chromaticity coordinates in the CIE color theory can be derived first from the definition of the stimulus $X, Y$, and $Z$. That is,

$X=\int_{380}^{780} P R \bar{x} \mathrm{~d} \lambda, \quad Y=\int_{380}^{780} P R \bar{y} \mathrm{~d} \lambda, \quad Z=\int_{380}^{780} P R \bar{z} \mathrm{~d} \lambda$,

where $P$ is the spectral distribution of the light source, $R$ is the reflectance curve for a typical object, and $\bar{x}, \bar{y}$, and $\bar{z}$ represent the spectral response curve of a standard observer. ${ }^{32-34}$ For dot matrix holograms, $R$ must be modified to be the product of the color-dispersion curve of the grating pixel and the color-dispersion curve of the reflective material that covers the dot matrix hologram. The chromaticity coordinates $x$ and $y$ are then defined as

$$
x=X /(X+Y+Z), \quad y=Y /(X+Y+Z) .
$$




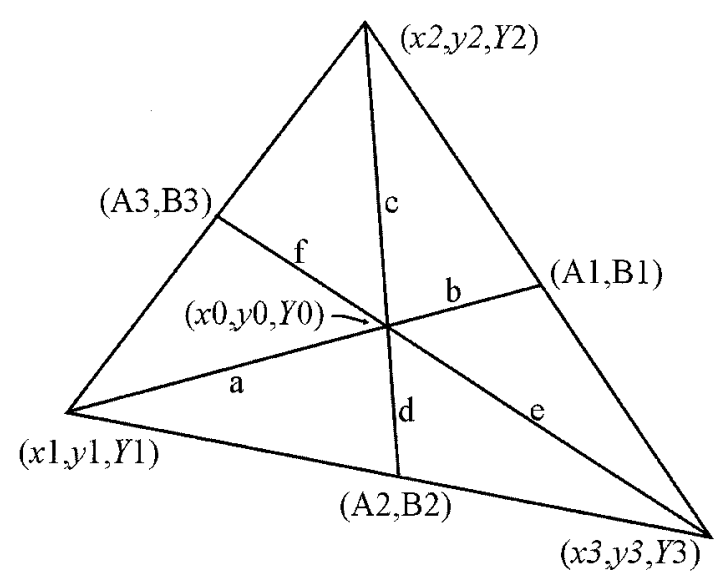

Fig. 17. Color composition by use of three extreme points located on the CIE color diagram.

Designating the desired graphic pixel that has chromaticity coordinates $(x 0, y 0)$ and sample luminance reflectance $Y 0$ as $[(x 0, y 0), Y 0]$, we can construct this graphic pixel by using three chosen grating pixels. ${ }^{35}$ Consider the case when the chromaticity coordinates of these three prespecified grating pixels are $(x 1, y 1)$, $(x 2, y 2)$, and $(x 3, y 3)$ and the diffractive light-beam intensity that can be generated by each of these three extreme points is expressed as $Y 1, Y 2$, or $Y 3$ (Fig. 17). The linearity of the CIE color theory thus yields

$$
\begin{aligned}
& x 0=u \times x 1+v \times x 2+w \times x 3, \\
& y 0=u \times y 1+v \times y 2+w \times y 3,
\end{aligned}
$$

where $u=b /(a+b), v=d /(c+d), w=f /(e+f), a=$ $\left[(x 1-x 0)^{2}+(y 1-y 0)^{2}\right]^{1 / 2}, b=\left[(A 1-x 0)^{2}+(B 1-\right.$ $\left.y 0)^{2}\right]^{1 / 2}, c=\left[(x 2-x 0)^{2}+(y 2-y 0)^{2}\right]^{1 / 2}, d=[(A 2-$ $\left.x 0)^{2}+(B 2-y 0)^{2}\right]^{1 / 2}, e=\left[(x 3-x 0)^{2}+(y 3-y 0)^{2}\right]^{1 / 2}$, and $f=\left[(A 3-x 0)^{2}+(B 3-y 0)^{2}\right]^{1 / 2}$. In addition, parameters $(A 1, B 1),(A 2, B 2)$, and $(A 3, B 3)$ can be shown to be

$$
\begin{aligned}
& A 1=\left(M_{32} x_{2}-M_{10} x_{1}-y_{2}+y_{1}\right) /\left(M_{32}-M_{10}\right), \\
& B 1=M_{32}\left(A_{1}-x_{2}\right)+y_{2}, \\
& A 2=\left(M_{31} x_{1}-M_{20} x_{2}-y_{3}+y_{2}\right) /\left(M_{31}-M_{20}\right), \\
& B 2=M_{31}\left(A_{2}-x_{3}\right)+y_{3}, \\
& A 3=\left(M_{21} x_{1}-M_{30} x_{3}-y_{1}+y_{3}\right) /\left(M_{21}-M_{30}\right), \\
& B 3=M_{21}\left(A_{3}-x_{1}\right)+y_{1},
\end{aligned}
$$

where $M_{32}$ is the slope between $(x 3, y 3)$ and $(x 2, y 2)$, $M_{21}$ is the slope between $(x 2, y 2)$ and $(x 1, y 1)$, etc.

The above discussion indicates the need for an algorithm with which to construct a specific color. This algorithm also stresses that, in designing a graphic pixel, the diffractive intensity of each grating pixel must be different even though all three grating pixels are under the same light source. To handle this issue more clearly, we use $[(x, y), Y]$ to represent the sample color, where $(x, y)$ is the chromaticity coordinate and $Y$ is the luminance reflectance $Y$. In

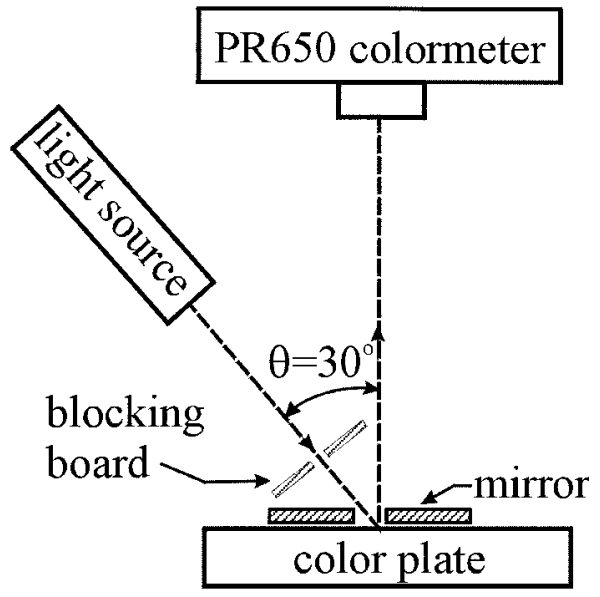

Fig. 18. Schematic of the experimental setup.

a dot matrix hologram approach, changing either the size of a grating pixel or using more than one grating pixel to represent that primary color can cause $Y$ to vary. In practical implementation, $Y$ can be a function of the exposure time or a function of the number of grating pixels. The microscopic photos of graphic pixels shown in Fig. 16 have different $Y$ values, even when the dpi values are identical.

With the flexibility provided by the newly developed dot matrix holographic printer, grating pixels of various pitches and orientations can be created with ease. To fully capture the power of this holographic printer and to create three-dimensional true-color dot matrix holograms we must first examine the chromaticity coordinate of each grating pixel fabricated and observed under various conditions. A total of 49 grating pitches that can cover the entire CIE border with equal distance from the nearest grating pixel were chosen as the fundamental color elements in this section. The experimental setup implemented is shown in Fig. 18; a color plate made from 49 blocks created by Sparkle is at the heart of this experiment. Each of these 49 color blocks, which measures $5 \mathrm{~mm}$ by $5 \mathrm{~mm}$, is filled with grating pixels of a distinctive grating pitch, which correspond to a chromaticity coordinate that is located near the border of the CIE chromaticity diagram. We measured the chromaticity coordinate that corresponds to each color block by using the configuration shown in Fig. 18. The chromaticity coordinates of these 49 color blocks, which directly correspond to the grating pitch in this experiment, evenly span the borders of the CIE chromaticity diagram. In addition, the 49 color blocks that correspond to 49 different wavelengths were not chosen to be equally divided between wavelengths 380 and $780 \mathrm{~nm}$ but to be equally divided between the CIE hoof-shaped boundary into 49 almost equally distant portions. More specifically, the diffracted light wavelength is determined directly from the linear dimension of the CIE chromaticity diagram, which means that the color presentation is linear in the CIE chromaticity diagram space instead of in 
Table 1. Characteristics of 6 of the 49 Color Blocks When the Incident Angle Equals $30^{\circ}$

\begin{tabular}{ccc}
\hline $\begin{array}{c}\text { Pitch } \\
\text { Number }\end{array}$ & $\begin{array}{c}\text { Wavelength } \\
(\mu \mathrm{m})\end{array}$ & $\begin{array}{c}\text { Pitch } \\
(\mu \mathrm{m})\end{array}$ \\
\hline 1 & 0.6270 & 1.2540 \\
2 & 0.5734 & 1.1468 \\
3 & 0.5356 & 1.0712 \\
4 & 0.5055 & 1.0110 \\
5 & 0.4904 & 0.9808 \\
6 & 0.4700 & 0.9400 \\
\hline
\end{tabular}

wavelength space. Table 1 lists 6 of the 49 grating pitches chosen. For configurations other than the one shown in Fig. 18 one can use the simple grating formula to determine the desired grating pitch and the corresponding color spectra. More specifically, if an observer wants to receive a specific color with wavelength at $380 \mathrm{~nm}$ when the incident light angle is $45^{\circ}$, the desired grating pitch can be calculated from the grating formula $d \sin \theta=n \lambda$. For $n=1$, the desired grating pitch will then be

$$
d(\mathrm{~nm})=\lambda / \sin \theta=380(\mathrm{~nm}) / \sin 45^{\circ} .
$$

A Photonics Research PR650 colormeter ${ }^{36}$ that acts as a spectrometer and can measure the incident light spectrum across the full visible color range every 4 $\mathrm{nm}$ was used to measure the light diffracted by the series of grating blocks located on the color plate. The light source was set such that the incident angle for each grating block under investigation was at $30^{\circ}$. The distance between the light source and the color plate was set long enough that the incident light appears collimated even when the light source is not a true collimated source. In addition, the color plate was covered with a reflective cover such that only the grating block under evaluation is illuminated by the light source. All other areas had the incident light reflected away from the entrance pupil of the PR650 colormeter. More specifically, two custom-made spatial filters were used to prevent scattering light and color noise from entering the colormeter. The first filter, a blocking board (Fig. 18) with a $10 \mathrm{~mm}$ by $10 \mathrm{~mm}$ hole located at its center, was used to limit the light incident onto the color plate. The second filter, a mirror with a $4 \mathrm{~mm}$ by $4 \mathrm{~mm}$ hole that was slightly smaller than the size of each color block, was used to ensure that only the light beam diffracted from the grating block under investigation entered the entrance pupil of the colormeter. Sandpaper instead of a mirror was also tried, with limited success, as the light beam scattered by the sandpaper appeared as background noise and reduced the color purity of the light beam diffracted by each grating block. This observation indicates that the color composition by diffraction gratings must be made with care such that one must consider the substrate reflectivity, surface roughness, etc. to obtain the best results.

During measurements, the color plate was moved horizontally on the $X-Y$ plane. The light beam diffracted from each grating block was measured block

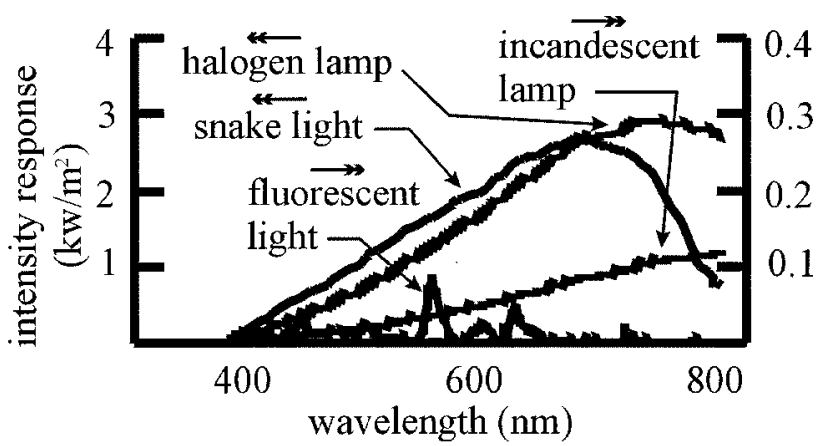

Fig. 19. Color spectra of the light sources used, where the intensity response is measured in kilowatts per square meter. The intensities of the halogen lamp and the snake light are taken from the left-hand axis; those of the incandescent lamp and fluorescent light, from right-hand axis.

by block to yield all 49 data points. The chromaticity coordinate and the diffracted spectrum from each block were retrieved directly from the colormeter. Experiments were performed with several widely available light sources including a halogen lamp, an incandescent light, a snake light, and a fluorescent light. We obtained the spectra of the light beams illuminated by these four light sources (Fig. 19) by projecting these light sources onto a white diffusive paper; then we used the colormeter to measure the scattered spectra. Note that the area of white paper illuminated by the light source was limited to the same area as that of the color block to ensure a fair comparison. More specifically, using the same $4 \mathrm{~mm}$ by $4 \mathrm{~mm}$ measurement area to measure both the diffracted light beam intensity and the scattered light intensity provided us with the same $Y$ value needed for our color composition studies. The spectra data shown in Fig. 19 indicate that all four light sources are biased toward the red portion of the visible spectrum. It is clear from Eqs. (3) that the chromaticity data of each grating block measured with these four light sources will be biased toward the red region. More specifically, substituting the data shown in Fig. 19 into Eqs. (3) clearly indicates that color blocks associated with the long-wavelength region will have chromaticity coordinates that will be located nearer the CIE boundary than those of the short-wavelength region. As the chromaticity coordinates of all 49 color blocks were found to appear within the CIE diagram in a continuous manner, only chromaticity coordinates measured from 6 of the 49 color blocks are shown in Fig. 20. The data confirmed the expectations. Note that the chromaticity coordinate that corresponds to the color block that represents the longest wavelength may move toward the center of the CIE diagram. The underlying reason for this is that the wavelength of the diffracted spectrum that can transmit onto the observer for that particular color block will correspond to a wavelength outside the perceivable range of the human eye, such as in the infrared region. From Fig. 20 it is clear that color blocks illuminated by different light sources will 


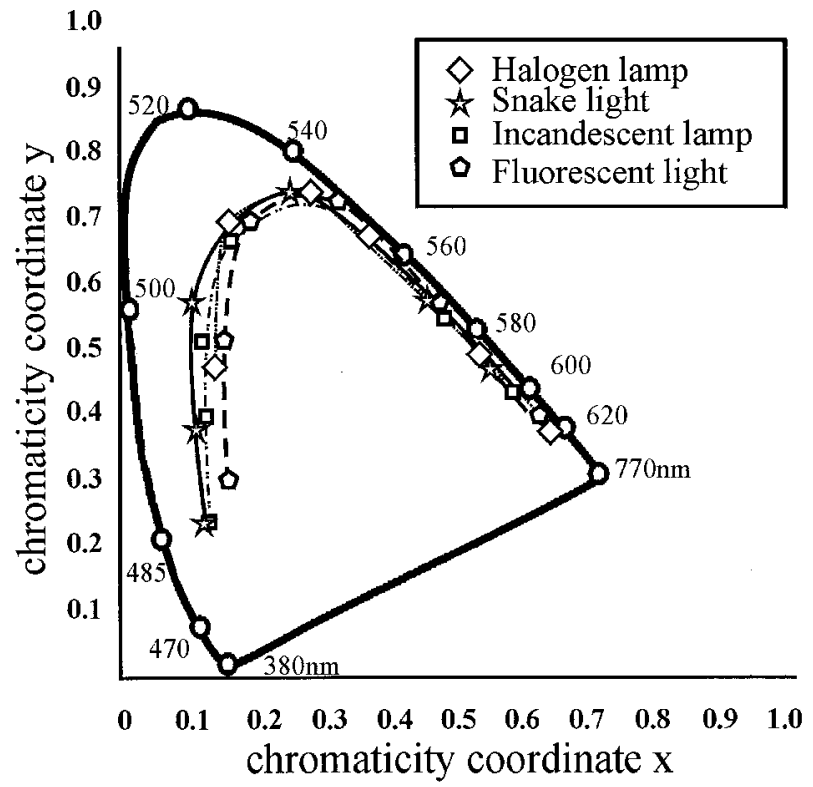

Fig. 20. Chromaticity coordinates of six color blocks illuminated by using four different light sources.

possess different chromaticity coordinates. That is, when grating pixels are used to compose colors, some of the colors can be reconstructed correctly only when specific light sources are used, whereas some colors can be reconstructed by use of all kinds of light sources. This observation provides us with an even more appealing approach that can be adapted for anticounterfeiting applications. When a light source with the appropriate spectral curve is chosen, the 49 color blocks can encompass almost the full CIE chromaticity diagram (Fig. 8).

\section{True-Color Three-Dimensional Dot Matrix Holograms}

As our perspective of object depth comes primarily from difference in viewing angle of our two eyes, the diffraction effect generated by each grating pixel can certainly be used to create three-dimensional (3D) perspectives of images in a two-dimensional medium. Furthermore, the CIE chromaticity diagram concept mentioned above, which uses multiple grating pixels to create a graphic pixel, can be used to create truecolor diffractive images. By combining the characteristics of the newly developed dot matrix holographic printer and the two concepts mentioned above, we can achieve the methodologies needed for implementing true-color 3D dot matrix holograms, as we detail below.

We take the three objects shown in Fig. 21 as examples. The triangle stands at the frontmost position, with the circle and the rectangle located behind it in that order. The left, middle, and right views of this set of three objects are shown in Fig. 21. This figure clearly indicates that the human perspective of object depth comes from the shifting of objects at different views. A special grating pixel located in the $x-y$ plane that has pitch $d$ and angle $\Omega$ between

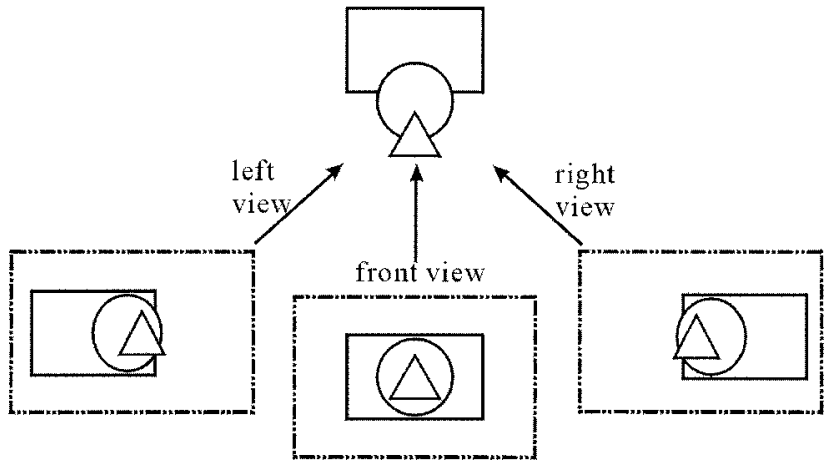

Fig. 21. Perspectives of 3D images at three different angles.

the grating orientation and the $x$ axis (Fig. 22) can be used as the vehicle to create the 3D image. The incident light beam propagates in the $y-z$ plane and has an angle $\gamma_{1}$ with the $z$ axis, i.e., $\alpha_{1}=\pi / 2$ and $\beta_{1}=$ $\pi / 2-\gamma_{1}$. The diffracted light beam travels along a direction that has angles $\alpha_{2}, \beta_{2}$, and $\gamma_{2}$ with respect to the $x, y$, and $z$ axes, respectively. In this case the equations that govern the relationship between the incident and the diffracted light beams can be derived from Eqs. (1) and (2) as follows:

$$
\begin{aligned}
& d=\frac{\lambda}{\left(\sin ^{2} \gamma_{1}+\sin ^{2} \gamma_{2}+2 \sin \gamma_{1} \sin \gamma_{2} \cos \omega\right)^{1 / 2}}, \\
& \Omega=\sin ^{-1}\left[\frac{\sin \omega}{\left(1+\frac{\sin ^{2} \gamma_{1}}{\sin ^{2} \gamma_{2}}+\frac{2 \cos \omega \sin \gamma_{1}}{\sin \gamma_{2}}\right)^{1 / 2}}\right], \\
& \omega=\tan ^{-1}\left(\frac{\cos \beta_{2}}{\cos \alpha_{2}}\right)+\frac{\pi}{2},
\end{aligned}
$$

where $\lambda$ is the wavelength of the light beam, $\Omega$ is positive if it is measured counterclockwise about the positive $z$ axis from the positive $x$ axis, and the value $\Omega$ shown in Eq. (12) takes the same sign as the value of $\cos \alpha_{2}$.

Considering the condition when a 3D dot matrix hologram is viewed at a distance of $300 \mathrm{~mm}$ and the

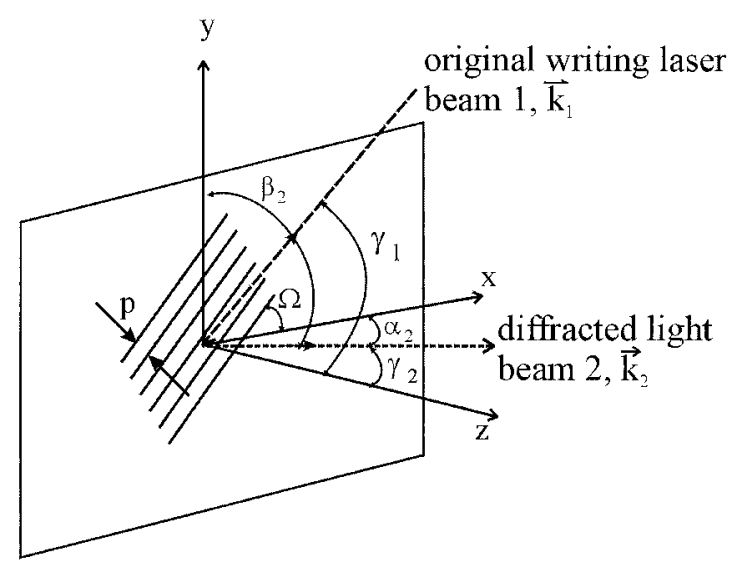

Fig. 22. Grating pixel for a true-color 3D dot matrix hologram. 


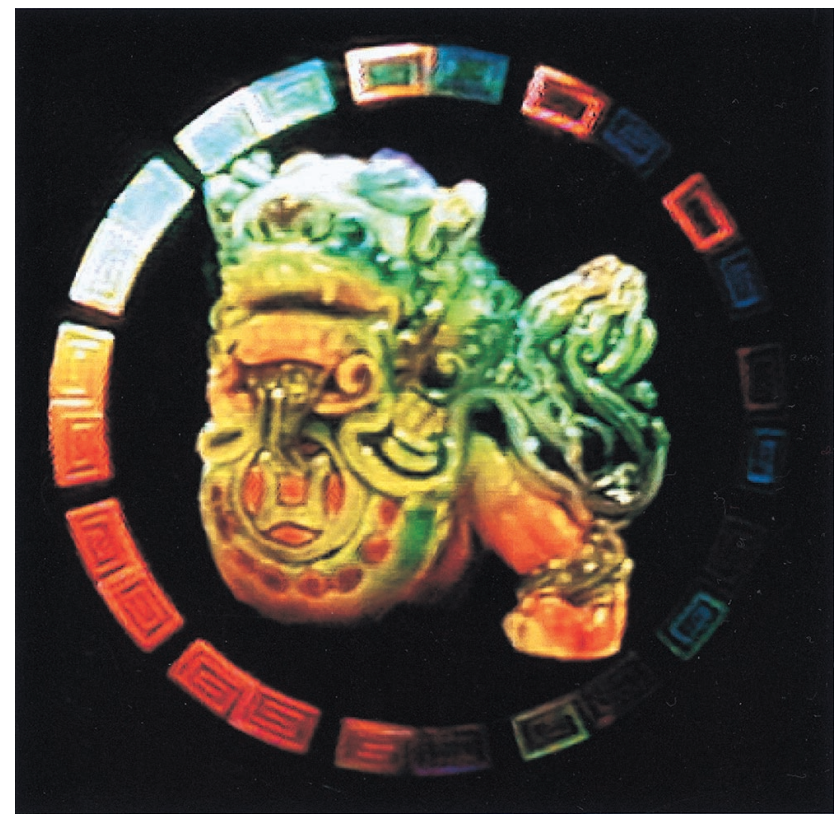

(a)

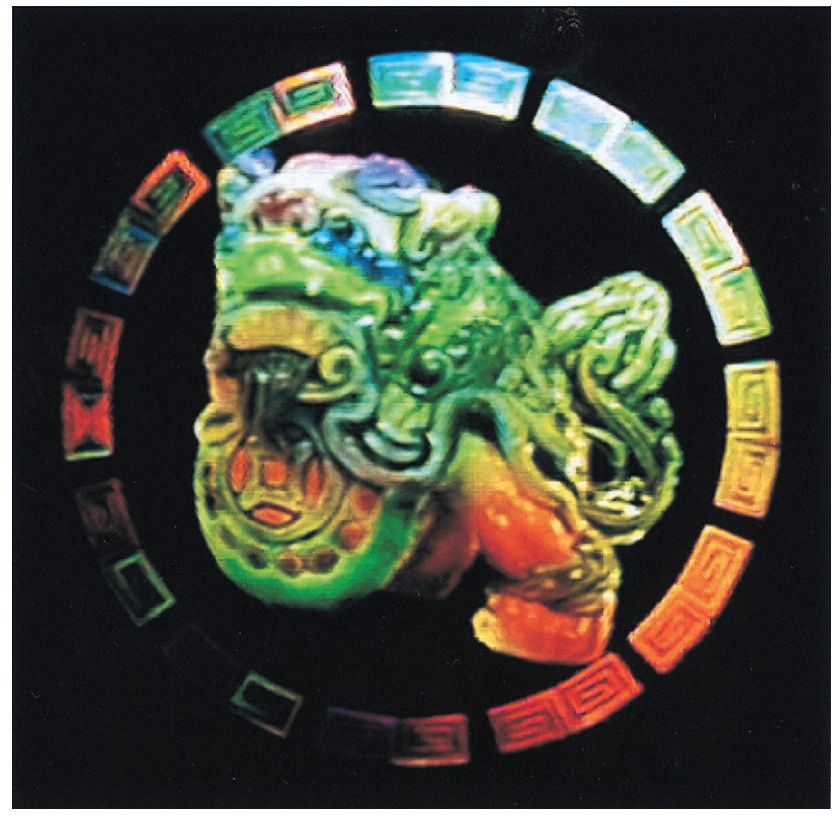

(b)

Fig. 23. (a) Left and (b) right images of a 3D true-color dot matrix hologram.

typical distance between an individual's two eyes is $70 \mathrm{~mm}$, the viewing angle difference between the two eyes will then be equal $\sim 13.3^{\circ}\left[=2 \tan ^{-1}(70 / 2 / 300)\right]$. More specifically, one needs to design the diffracted light by making the grating plane angle $6.65^{\circ}$ $\left[=\tan ^{-1}(70 / 2 / 300)\right]$ for the right eye and $-6.65^{\circ}$ $\left[=-\tan ^{-1}(70 / 2 / 300)\right]$ for the left eye with respect to the $z$ axis (angle $\beta_{2}$ ). In a 3D dot matrix hologram, each point of the hologram may be used to represent a different depth perspective. For example, if a background image point is to be placed $10 \mathrm{~mm}$ behind the hologram, the background image point must be shifted $1.17 \mathrm{~mm}[=10(70 / 2 / 300)]$ at the two views for both the left and the right eyes. In other words, the background image must shift leftward $1.17 \mathrm{~mm}$ for the left view and rightward $1.17 \mathrm{~mm}$ for the right view. In addition, for foreground and background image points of an equal depth perspective, the two views must be shifted by equal distances but in opposite directions.

The above discussion indicates that, to create a 3D dot matrix hologram, the designer may first create pseudo-3D models in a computer and then take views horizontally, say, every $13.3^{\circ}$. This can be an extremely time-consuming process if a delicate 3D model is to be created with a computer. The method described in this paper is somewhat simpler and more practical. Several digital cameras were used to take pictures of a real object from various perspectives. These pictures, which represent different perspectives of the real object, are then used to create a $3 \mathrm{D}$ dot matrix hologram of the real object. This process involves integrating the effects of multiple grating pixels and then re-creating each true-color graphic pixel at a different perspective. Figure 23 shows the left and right pictures a hologram, which is the $3 \mathrm{D}$ image of a ceramic lion. Even though this 3D true-color effect cannot be created without a dot matrix hologram, one can reconstruct the stereographic effect by using the so-called parallel method. That is, if one lets his or her eyes see the two images simultaneously by focusing at infinity, the 3D effect will be re-created. It can be seen clearly from Fig. 23 a 3D true-color dot matrix hologram easily can made by use of the newly developed dot matrix holographic printer.

\section{Conclusions and Discussion}

The optical configuration of the newly developed holographic printer Sparkle detailed in this paper clearly demonstrates a simple yet efficient algorithm for controlling the pitch of each grating pixel, which in turn determines the color to be seen by a viewer at a specific location. With the introduction of such capabilities into a dot matrix holographic mastering system, true-color dot matrix holograms are now a reality. From the theoretical discussions, it is clear that positions of the light source and the observer, the grating pixel, the spectral response of light source, the pitch and orientation of each grating pixel, the composition of each graphic pixel, etc. must all be specified to achieve the effects intended by the graphic designer. With the capability to vary the grating pitch now easily available, creating specific visual effects in a dot matrix hologram is practical and easy. In addition, a good preview function will now become available because all parameters needed to make a sound grating formula calculation are now fully controllable in the holographic printer described here. The experimental data obtained have clearly demonstrated that composing color by using grating pixels of various pitches can encompass almost the 
whole CIE chromaticity diagram. Grating pixels can be used to give us a much wider color range than any color-composition schemes currently available; many colors that were typically unattainable by more-traditional means, such as metallic colors, can now be re-created by the newly developed dot matrix holographic printer.

This research was supported partially by the National Science Council of Taiwan under contracts NSC 85-2622-E-002-017R and NSC 86-2622-E-002023R.

\section{References}

1. D. Gabor, "A new microscope principle," Nature (London) 161, 777-778 (1948).

2. D. Gabor, "Microscope by reconstructed wave-fronts," Proc. R. Soc. London Ser. A 197, 454-487 (1949).

3. E. N. Leith and J. Upatnieks, "Wavefront reconstruction with diffused illumination and three-dimensional objects," J. Opt. Soc. Am. 54, 1295-1301 (1964).

4. B. R. Brown and A. W. Lehman, "Complex spatial filtering with binary masks," Appl. Opt. 5, 967-969 (1966).

5. B. Lesem, P. M. Hirsch, and A. Jordan, "Computer synthesis of holograms or 3-D display," Commun. ACM 11, 661-674 (1968).

6. M. Nakajima and Y. Mitsuhashi, "Computer-generated polarization holography: automatic hologram making system and the quality of the reconstructed image," in International Conference on Computer-Generated Holography, S. H. Lee, ed., Proc. SPIE 437, 79-88 (1983).

7. F. Wyrowski, R. Hauck, and O. Bryngdahl, "Computer holography: object dependent deterministic diffusers," Opt. Commun. 63, 81-84 (1987).

8. S. A. Benton, "The reflection alcove hologram: a computergraphic holographic stereogram," in Computer-Generated Holography II, S. H. Lee, ed., Proc. SPIE 884, 106-113 (1988).

9. R. Stephen, "Enhanced nondestructive holographic reconstruction," U.S. patent 4,953,924 (4 September 1990).

10. W. Lehman and J. Ojeda-Castaneda, "Computer generated holography: novel procedure," Opt. Commun. 3, 181-184 (1993).

11. R. G. Dorsche, A. W. Lehman, and S. Sinzinger, "Fresnel pingpong algorithm for two-plane computer-generated holograms display," Appl. Opt. 33, 846-875 (1994).

12. U. Schnars and W. P. O. Jüeptner, "Digital recording and reconstruction of holograms in hologram interferometry and shearography," Appl. Opt. 33, 4373-4377 (1994).

13. E. Zhang, S. Noehte, C. Dietrich, and R. Maenner, "Gradual and random binarization of gray-scale holograms," Appl. Opt. 34, 5987-5995 (1995).

14. L. Cai, "Generation of 3D image from computer data with dot array rainbow hologram," in Holographic Optical Elements and Display, F. S. Lin and D. Xu, eds., Proc. SPIE 2885, 17-25 (1996).

15. T. Wang, Y. Li, S. Yang, S. Fan, S. Zhang, and H. Wen, "Fractal IFS in laser lithographic hologram," J. Optoelectron. Laser Suppl. 9, 397-399 (1998).

16. S. Takahashi, "Method for producing a display with a diffraction grating pattern and a display produced by the method," U.S. patent 5,058,992 (22 October 1991).
17. S. Takahashi, T. Toda, and F. Iwata, "Method of manufacturing display having diffraction grating patterns," U.S. patent 5,132,812 (21 July 1992).

18. F. Davis, "Holographic image conversion method for making a controlled holographic grating," U.S. patent 5,262,879 (16 November 1993).

19. C. Newswanger, "Holographic diffraction grating patterns and method for creating the same," U.S. patent 5,291,317 (1 March 1994).

20. F. Davis, "System for making a hologram of an image by manipulating object beam characteristics to reflect image data," U.S. patent 5,822,092 (13 October 1998).

21. W. J. Wu, C. K. Lee, C. T. Hsieh, S. J. Chiang, S. T. Lin, C. C. Hsu, C. C. Lin, and C. W. Kuo, "Methods to create true-color dot matrix holograms," U.S. patent pending (application filed 25 June 1997).

22. L. E. Drain, The Laser Doppler Technique (Wiley, New York, 1980).

23. R. L. van Renesse, Optical Document Security (Artech House, Norwood, Mass., 1994).

24. M. J. Verheijen, "E-beam lithography for digital holograms," J. Mod. Opt. 40, 711-721 (1993).

25. N. Yoshikawa, M. Itoh, and T. Yatagai, "Binary computergenerated holograms for security applications from a synthetic double-exposure method by electron-beam lithography," Opt. Lett. 23, 1483-1485 (1998).

26. T. Hamano and H. Yoshikawa, "Image-type CGH by means of e-beam printing," in Practical Holography XII, S. A. Benton, ed., Proc. SPIE 3293, 170-180 (1998).

27. J. H. Huang, S. L. Yeh, C. K. Lee, and T. K. Huang, "Large format grating image hologram based on e-beam lithography," in Practical Holography X, S. A. Benton, ed., Proc. SPIE 2652, 117-123 (1996).

28. M. R. Chamberlain, "Elemental analysis of kinematic optically variable devices," in Optical Security and Counterfeit Deterrence Techniques, R. L. van Renesse, ed., Proc. SPIE 2659, 170-180 (1996).

29. D. M. Newman, R. W. Hawley, D. L. Goeckel, R. D. Crawford, S. Abraham, and N. C. Gallagher, "Efficient storage, computation, and exposure of computer-generated holograms by electron-beam lithography,” Appl. Opt. 32, 2555-2565 (1993).

30. J. Fan, D. Zaleta, K. S. Urquhart, and S. H. Lee, "Efficient encoding algorithms for computer-aided design of diffractive optical elements by the use of electron-beam fabrication," Appl. Opt. 34, 2522-2533 (1995).

31. National Nano Device Laboratory, http://www.ndl.gov.tw/ (1999).

32. F. W. Billmeyer, Jr. and M. Saltzman, Principles of Color Technology (Wiley, New York, 1981).

33. G. Wyszecki and W. S. Stiles, Color Science: Concepts and Methods, Quantitative Data and Formulae (Wiley, New York, 1982).

34. R. W. G. Hunt, Measuring Colour (Ellis Horwood, Ashburton, Devon, UK, 1995).

35. C. W. Tu, Y. A. Han, C. K. Lee, W. J. Wu, A. S. T. Peng, E. H. Z. Liao, and J. T. Lee, "Expanding a color presentation range with true-color dot matrix holograms," in Practical Holography XIII, S. A. Benton, ed., Proc. SPIE 3637, 130-140 (1999).

36. Photo Research, Inc., PR-650 Spectra Colorimeter Operating Manual (Photo Research, Inc., Chatsworth, California, 1996). 